\title{
Complex magnetic phase diagram in noncentrosymmetric EuPtAs
}

\author{
W. Xie, ${ }^{1,2}$ S. S. Luo, ${ }^{1}$ H. Su, ${ }^{1}$ X. Y. Zheng, ${ }^{1}$ Z. Y. Nie, ${ }^{1}$ M. Smidman $\odot,{ }^{1, *}$ T. Takabatake $\odot,{ }^{1,3}$ and H. Q. Yuan ${ }^{1,4,5, \dagger}$ \\ ${ }^{1}$ Center for Correlated Matter and Department of Physics, Zhejiang University, Hangzhou 310058, China \\ ${ }^{2}$ Deutsches Elektronen-Synchrotron (DESY), Notkestrasse 85, 22607 Hamburg, Germany \\ ${ }^{3}$ Department of Quantum Matter, Graduate School of Advanced Science and Engineering, \\ Hiroshima University, Higashi-Hiroshima 739-8530, Japan \\ ${ }^{4}$ Collaborative Innovation Center of Advanced Microstructures, Nanjing 210093, China \\ ${ }^{5}$ State Key Laboratory of Silicon Materials, Zhejiang University, Hangzhou 310058, China
}

(Received 23 July 2021; revised 28 October 2021; accepted 8 November 2021; published 18 November 2021)

\begin{abstract}
We report the observation of multiple magnetic transitions in single crystals of EuPtAs using magnetization, transport, and thermodynamic measurements. EuPtAs crystallizes in the noncentrosymmetric tetragonal LaPtSitype structure (space group $I 4_{1} m d$ ) and undergoes a second-order antiferromagnetic (AFM) transition at $14.8 \mathrm{~K}$, which is followed by a first-order transition at about 7.5 K. Both transitions are suppressed by magnetic fields applied parallel and perpendicular to the $c$ axis, and multiple field-induced transitions are observed for both field directions, leading to complex temperature-field diagrams with a domelike magnetic phase for fields parallel to the $c$ axis. Moreover, the dependence of the low-temperature resistivity on the application of a training field, together with a lack of remanent magnetization, suggests the presence of multiple AFM domains in zero field.
\end{abstract}

DOI: 10.1103/PhysRevB.104.174425

\section{INTRODUCTION}

Magnets with a noncentrosymmetric structure often exhibit complex magnetic structures due to the presence of the Dzyaloshinskii-Moriya (DM) interaction [1,2]. Unlike other exchange interactions which typically favor collinear spin structures, the DM interaction tends to orientate the spins perpendicular to each other. The competition between these competing tendencies can lead to noncollinear magnetic structures which could be topologically nontrivial in real space [3]. For example, a magnetic skyrmion phase with a topologically nontrivial spin texture has been experimentally discovered in $\mathrm{MnSi}$, which has the chiral B20-type structure (space group $P 2{ }_{1} 3$ ) [4-6]. Subsequently, a series of additional compounds with the B20 structure have also been identified to host magnetic skyrmions, such as $\mathrm{Fe}_{1-x} \mathrm{Co}_{x} \mathrm{Si}[7,8]$, FeGe $[9,10]$, and $\mathrm{Cu}_{2} \mathrm{OSeO}_{3}[11,12]$. Recently EuPtSi, which also crystallizes in a chiral cubic structure (LaIrSi-type, space group $P 2{ }_{1} 3$ ) [13], was shown to be an unusual example of an $f$-electron system hosting magnetic skyrmions [14-16], where the size of a single skyrmion for $H \|[111]$ is only $18 \AA$, 10 times smaller than those in $\mathrm{MnSi}$ [17]. In addition to these compounds with chiral structures, it was found that compounds with a noncentrosymmetric polar structure are also promising candidates for realizing skyrmions, such as $\mathrm{GaV}_{4} \mathrm{~S}_{8}$ with a lacunar spinel structure [18], which hosts a Néel-type skyrmion phase. For CeAlGe with a polar tetragonal structure, evidence for a meron/antimeron phase has been reported, which has a double- $k$ spin structure with a topological charge $Q=1 / 2$

*Corresponding author: msmidman@zju.edu.cn

${ }^{\dagger}$ Corresponding author: hqyuan@zju.edu.cn
[19], in contrast to a skyrmion lattice which has a triple- $k$ structure with $Q=1$.

Although quite a few types of materials have been found to host skyrmionlike topological spin textures, such explorations in rare-earth compounds are still relatively limited after the report of EuPtSi, with the focus on some Gd-based centrosymmetric compounds where magnetic skyrmions with reduced size were commonly revealed in the absence of the DM interaction [20-23]. Here we focus on the Eu-based compound EuPtAs. Unlike other reported members in the EuTX series [24], EuPtAs crystallizes in the polar tetragonal LaPtSi-type structure (space group $I 4_{1} \mathrm{md}$ ) [25] that is isostructural to $\mathrm{CeAlGe}$. The crystal structure is displayed in the inset of Fig. 1. For the Eu atoms, the nearest-neighbor distance of $4.23 \AA$ (in plane) is very close to the next-nearest-neighbor distance $4.28 \AA$ (out of plane), which are also comparable to the $\mathrm{Ce}-\mathrm{Ce}$ distances in CeAlGe. However, in comparison to $\mathrm{Ce}^{3+}$ in $\mathrm{CeAlGe}$, the Hund's rule ground state of $\mathrm{Eu}^{2+}$ in EuPtAs has a total orbital angular momentum $L=0$, which offers the opportunity to examine the magnetic properties in the same crystal structure but with greatly reduced magnetocrystalline anisotropy. On the other hand, the magnetic atoms in both compounds form nearly equilateral triangular sublattices where magnetic frustration is possible, which has been proposed to be another important mechanism for the realization of magnetic skyrmions, even in centrosymmetric compounds [20].

In this paper, we report magnetization, transport, and thermodynamic measurements of EuPtAs single crystals grown by a $\mathrm{Pb}$-flux method, revealing that EuPtAs undergoes two magnetic transitions in zero field at 14.8 and $7.5 \mathrm{~K}$, which are both continuously suppressed in applied magnetic fields up to $9 \mathrm{~T}$. We construct the magnetic field-temperature $(H-T)$ phase diagrams for magnetic fields applied parallel and 


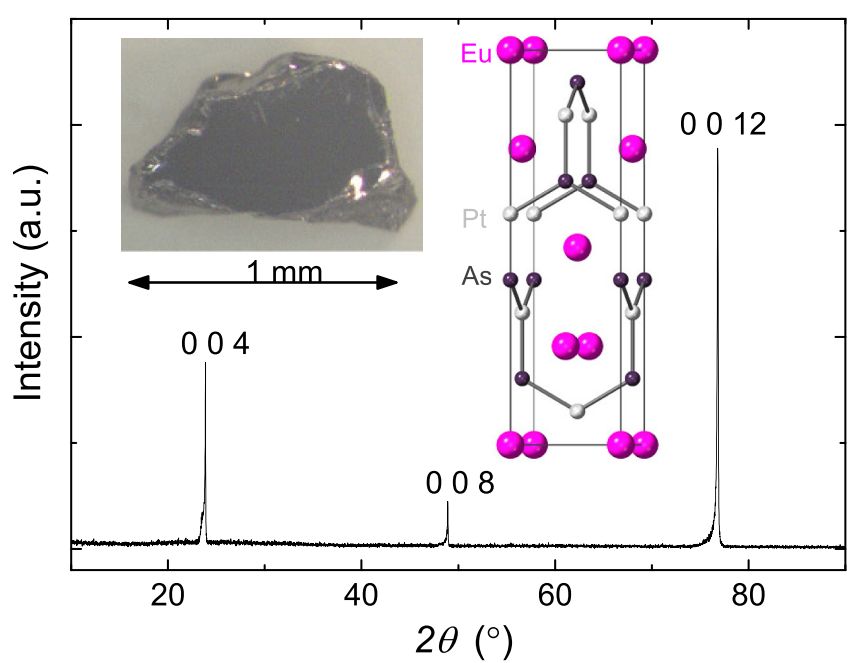

FIG. 1. XRD pattern of a EuPtAs single crystal with a surface plane perpendicular to the tetragonal $c$ axis. The left inset shows a photograph of a piece of single crystal with a typical length of $1 \mathrm{~mm}$. The unit cell of EuPtAs is shown in the right inset.

perpendicular to the easy $c$ axis, featuring a number of fieldinduced magnetic phases.

\section{EXPERIMENTAL METHODS}

Single crystals of EuPtAs were grown using a $\mathrm{Pb}$-flux method. Eu pieces (99.9\%), Pt powder $(99.9+\%)$, and As grains $(99.999 \%)$ from Alfa Aesar were mixed in an offstoichiometric ratio and put into an alumina crucible together with excessive $\mathrm{Pb}$ ingots $(99.99+\%)$. The crucible was sealed in an evacuated quartz ampule, which was slowly heated to $1100^{\circ} \mathrm{C}$ and kept there for 2 days before being cooled at a rate of $3^{\circ} \mathrm{C} / \mathrm{h}$ to $750^{\circ} \mathrm{C}$, at which temperature the ampule was removed from the furnace and centrifuged. Shiny platelike crystals with typical dimensions of $1 \times 0.5 \times 0.2 \mathrm{~mm}^{3}$ were obtained. In order to remove the residual $\mathrm{Pb}$ flux, the crystals were etched in dilute hydrochloric acid and polished. A photograph of a piece of single crystal is shown in the inset of Fig. 1. The crystal structure and orientation were characterized by x-ray diffraction (XRD) using a PANalytical X'Pert MRD diffractometer with $\mathrm{Cu} \mathrm{K}_{\alpha 1}$ radiation monochromated by graphite. The chemical composition was determined by energy-dispersive x-ray spectroscopy (EDS) using a Hitachi SU-8010 field emission scanning electron microscope. The electrical resistivity and heat capacity were measured using a Quantum Design Physical Property Measurement System (QD PPMS-9T) with a ${ }^{3} \mathrm{He}$ insert. The magnetic susceptibility and magnetization were measured down to $2 \mathrm{~K}$ using a Quantum Design Magnetic Property Measurement System (QD MPMS-5T) and a vibrating sample magnetometer on a Physical Property Measurement System (PPMS-14T).

\section{RESULTS AND DISCUSSIONS}

\section{A. Zero-field characterization}

Figure 1(a) shows the XRD pattern measured on a piece of plate-shaped single crystal, in which the diffraction peak

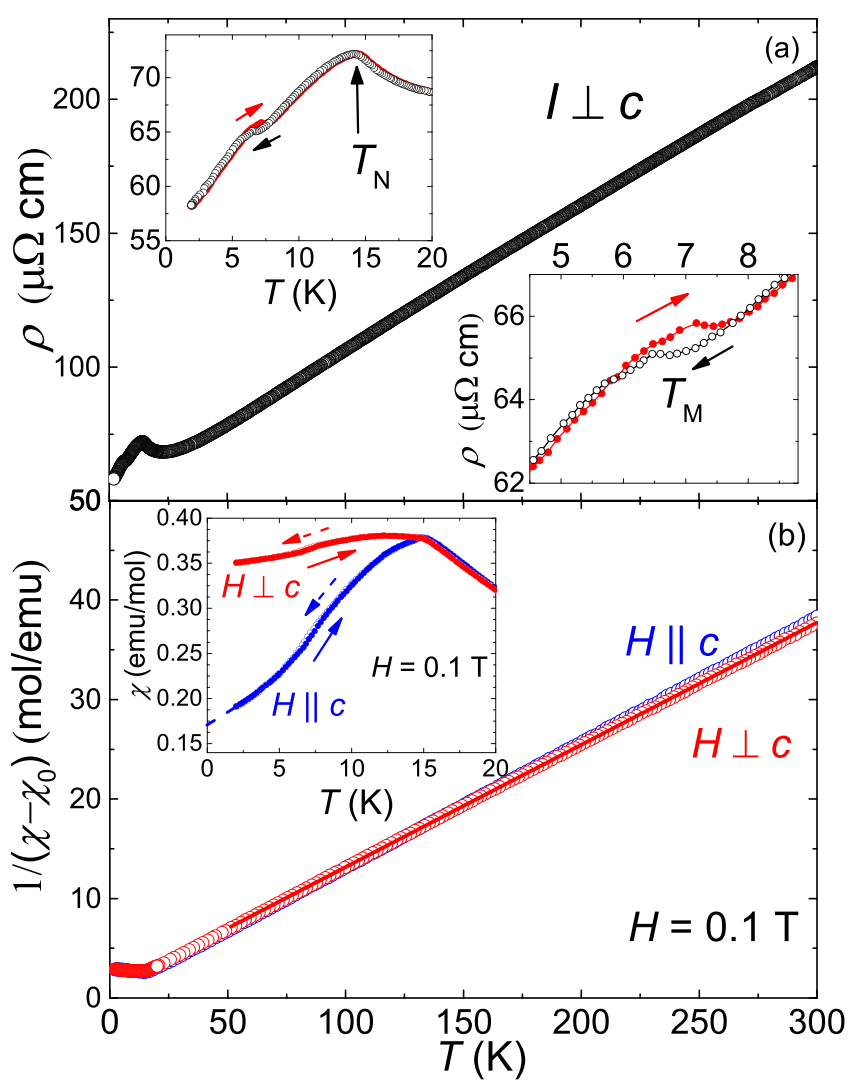

FIG. 2. (a) Temperature dependence of the resistivity $\rho(T)$ in the temperature range $300-1.9 \mathrm{~K}$. The insets display low-temperature enlargements in order to show the transitions at $T_{\mathrm{N}}$ and $T_{\mathrm{M}}$. (b) Inverse magnetic susceptibility from 300 to $2 \mathrm{~K}$ for both $H \| c$ and $H \perp c$ measured in an applied field of $0.1 \mathrm{~T}$. The solid lines are fits of the high-temperature data with the modified Curie-Weiss law. The inset shows the low-temperature part of $\chi(T)$ for both directions.

positions match well with the expected $(00 l)(l=$ integer $)$ peak positions for EuPtAs [25], indicating that the platelike surface is oriented perpendicular to the $c$ axis. The EDS measurements performed at multiple positions on the crystal show an average atomic ratio of 1:1.02:1.01 for Eu:Pt:As, which is very close to the stoichiometric EuPtAs composition.

The temperature dependence of the electric resistivity $\rho(T)$, measured with a current $I \perp c$, is shown in the main panel of Fig. 2(a). The nearly linear behavior in $\rho(T)$ is commonly observed for $\mathrm{Eu}^{2+}$-based intermetallics [26,27], which is generally ascribed to arising from the electron-phonon scattering [27,28]. At low temperature, $\rho(T)$ shows a peak at the magnetic ordering temperature $T_{\mathrm{N}} \approx 14.8 \mathrm{~K}$ and a cusp at $T_{\mathrm{M}}=6.5 \mathrm{~K}$ on cooling $(7 \mathrm{~K}$ on warming). While negligible differences are found at $T_{\mathrm{N}}$ between the cooling and warming processes, hysteresis is observed at $T_{\mathrm{M}}$, as shown in the insets of Fig. 1(a).

The temperature dependence of the inverse magnetic susceptibility is displayed in the main panel of Fig. 2(b), where the high-temperature behavior can be well described by the modified Curie-Weiss form $\chi=\chi_{0}+C /\left(T-\theta_{P}\right)$ with an effective magnetic moment of $\mu_{\text {eff }}=7.95 \mu_{B} / \mathrm{Eu}$ and a Weiss temperature $\theta_{P}=-4.7 \mathrm{~K}$ for $H \| c$, while for $H \perp c, \quad \mu_{\text {eff }}=8.03 \mu_{B} / \mathrm{Eu}$ and $\theta_{P}=-7.3 \mathrm{~K}$. For 
both directions there is a temperature-independent susceptibility $\chi_{0}=0.004 \mathrm{emu} / \mathrm{mol}$. The magnitude of $\mu_{\mathrm{eff}}$ is close to the expected value of $7.94 \mu_{B}$ for the well-localized $\mathrm{Eu}^{2+}$ electronic state with $J=7 / 2$. The inset of Fig. 2(b) displays the low-temperature $\chi(T)$ for both $H \| c$ and $H \perp c$, where a cusp is clearly observed at $T_{\mathrm{N}}$, while a weak slope change with small thermal hysteresis occurs at $T_{\mathrm{M}}$. The anisotropic behavior in $\chi(T)$ at $T \leqslant T_{\mathrm{N}}$ indicates an antiferromagnetic (AFM) transition with the $c$ axis as the easy axis. In addition, extrapolation of the $c$ axis susceptibility $\chi_{c}(T)$ down to zero temperature gives a finite $\chi_{c}(0)$ of about $0.168 \mathrm{emu} / \mathrm{mol}$, yielding the ratio $\chi_{c}(0) / \chi\left(T_{\mathrm{N}}\right)=$ 0.44 . This feature is inconsistent with a collinear AFM structure of the Eu spins along the $c$ axis, for which $\chi_{c}(0)$ should be zero [29]. A possible explanation is that the Eu spins tilt away from the $c$ axis, forming a noncollinear magnetic structure. Two consecutive magnetic transitions at low temperature are also observed in some other Eu-based intermetallics, for instance, $\mathrm{EuPt}_{2} \mathrm{As}_{2}$ [30] and $\mathrm{EuPd}_{2} \mathrm{As}_{2}$ [31]. While the spin structure change in $\mathrm{EuPt}_{2} \mathrm{As}_{2}$ is still not well understood, the spins in $\operatorname{EuPd}_{2} \mathrm{As}_{2}$ are reported to be mainly orientated in the $a b$ plane below $T_{\mathrm{N}}$, which cant toward the $c$ axis on crossing $T_{\mathrm{M}}$. Consequently, $\chi_{c}$ is flat between $T_{\mathrm{N}}$ and $T_{\mathrm{M}}$ and then decreases significantly below $T_{\mathrm{M}}$, while $\chi_{a b}$ decreases continuously below $T_{\mathrm{N}}$ with a smaller decreasing rate on crossing $T_{\mathrm{M}}$ [31]. However, both $\chi_{c}(T)$ and $\chi_{a b}(T)$ for EuPtAs decrease monotonically below $T_{\mathrm{N}}$ where $\chi_{c}(T)$ displays a steeper drop. In addition, the rate at which both $\chi_{c}(T)$ and $\chi_{a b}(T)$ decrease varies continuously with temperature, where there is an inflection point near $T_{\mathrm{M}}$. This is in contrast to that observed in $\mathrm{EuPt}_{2} \mathrm{As}_{2}$ [30] and $\mathrm{EuPd}_{2} \mathrm{As}_{2}$ [31], which might be due to a gradual spin canting with decreasing temperature.

The temperature dependence of the specific heat as $C(T) / T$ is shown in Fig. 3(a). A jump at about $14.9 \mathrm{~K}$ and two small peaks centered at $7.3 \mathrm{~K}$ (on warming) are observed. The former corresponds to the AFM transition at $T_{\mathrm{N}}$, while the latter peaks arise due to the magnetic transition at $T_{\mathrm{M}}$, where the difference between warming and cooling processes is consistent with $T_{\mathrm{M}}$ being a first-order transition. The magnetic entropy is shown in Fig. 3(b), which was calculated by integrating the electronic contribution $C_{\mathrm{e}} / T$ after subtracting the lattice contribution, where $C_{\mathrm{e}} / T$ was assumed to be linear in $T$ for $T \leqslant 0.4 \mathrm{~K}$. Note that due to the lack of a suitable nonmagnetic reference compound, the high-temperature phonon contribution was fitted with two models. The results are shown in the inset of Fig. 3(b), which indicates that a model combining both Debye and Einstein contributions $\left\{C_{L}(T)=(1-p) C_{D}(T)+p C_{E}(T)[27]\right\}$ can reasonably describe the experimental data at high temperatures, with fitted values of the Debye temperature $\Theta_{\mathrm{D}}=406 \mathrm{~K}$, the Einstein temperature $\Theta_{\mathrm{E}}=111 \mathrm{~K}$, and a weighting factor $p=0.66$. The entropy released at $T_{\mathrm{N}}$ is slightly less than $\mathrm{R} \ln 8$, the full entropy expected for $\mathrm{Eu}^{2+}$ with an eightfold degenerate ground-state multiplet $(J=7 / 2)$. The integrated magnetic entropy reaches $\mathrm{R} \ln 8$ at $20 \mathrm{~K}$ and then saturates for $T \geqslant$ $30 \mathrm{~K}$. The additional entropy above $20 \mathrm{~K}$ likely results from an incomplete subtraction of the phonon contribution [32]. A notable feature in $C_{\mathrm{e}} / T$ is a tail above $T_{\mathrm{N}}$. This could arise from magnetic fluctuations or short-range magnetic cor-

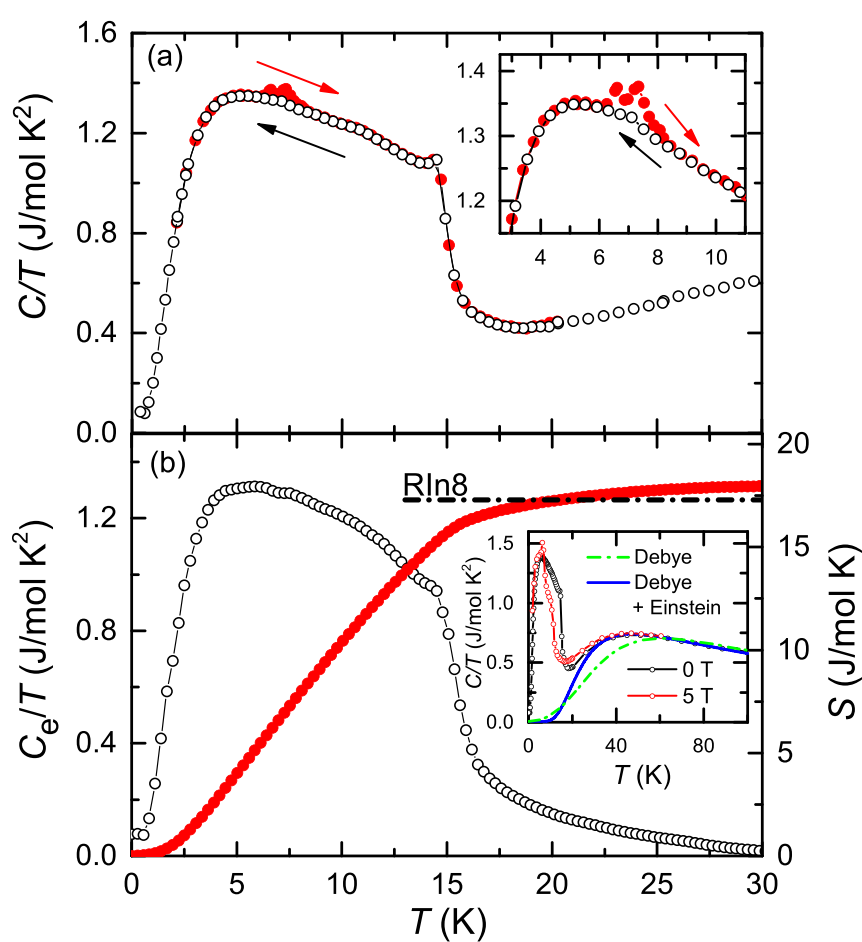

FIG. 3. (a) Low-temperature specific heat as $C(T) / T$ measured on warming and cooling, as indicated by the arrows. The inset enlarges the data to display the first-order transition at $T_{\mathrm{M}}$. (b) The magnetic entropy calculated (right-hand axis) by integrating the electronic contribution $C_{\mathrm{e}}(T) / T$ (left-hand axis). The inset shows the fits to the high-temperature data of $C(T) / T$ for two adopted models.

relations above $T_{\mathrm{N}}$, which seem to be further enhanced on applying a magnetic field of $5 \mathrm{~T}$, as shown in the inset of Fig. 3(b).

\section{B. In-field measurements}

Figures 4(a) and 4(b) show $\chi(T)$ for various magnetic fields applied along the $c$ axis and $a b$ plane, respectively. With increasing field, $T_{\mathrm{N}}$ is suppressed to lower temperatures with the corresponding peak becoming broader for both directions. $T_{\mathrm{M}}$ is also continuously suppressed with field and is no longer observed down to $1.9 \mathrm{~K}$ for $H \geqslant 3 \mathrm{~T}$. A field-induced transition (marked by the upward green arrows) is observed for $H \geqslant 3.5 \mathrm{~T}$ for $H \| c . \chi(T)$ exhibits a rather linear behavior down to the lowest temperature after crossing this transition (see the inset), which displays a nonmonotonic field dependence, first shifting to higher temperatures and then to lower temperatures with increasing field before disappearing above $6 \mathrm{~T}$. For $H \perp c$, the field-induced transition appears for $H \geqslant 5.5 \mathrm{~T}$, where there is an abrupt increase in $\chi(T)$ below the transition, together with thermal hysteresis. In addition, anomalies are also resolved above $T_{\mathrm{N}}$ for $H \geqslant 3.5 \mathrm{~T}$ (upward magenta arrows) for $H \perp c$, which become increasingly prominent at higher fields. These behaviors in $\chi(T)$ indicate multiple field-induced phases in EuPtAs.

To further track the transitions in EuPtAs, $\rho(T)$ and $C(T)$ were measured in applied magnetic fields up to $9 \mathrm{~T}$. Overall, the absolute value of $\rho(T, H)$ is suppressed in applied fields, where anomalies at the various phase transitions are also 


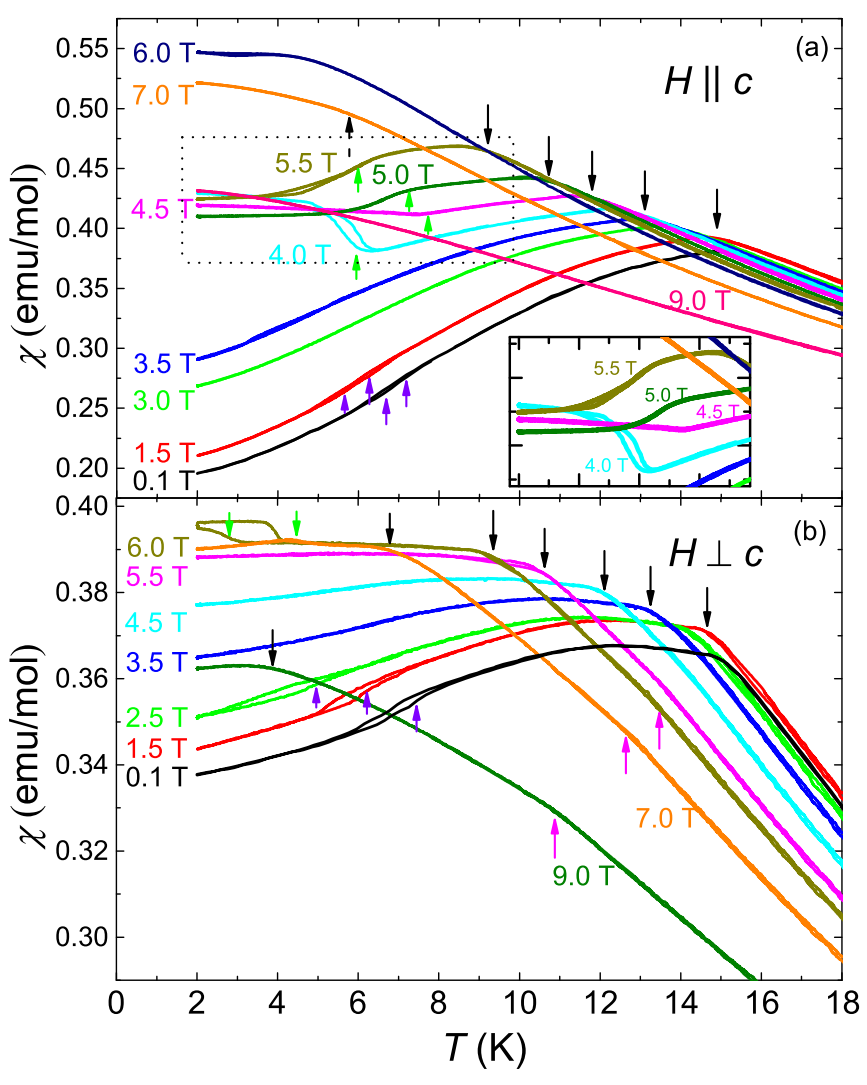

FIG. 4. Low-temperature magnetic susceptibility $\chi(T)$ in magnetic fields up to $9 \mathrm{~T}$ applied (a) parallel to and (b) perpendicular to the $c$ axis. The different-colored arrows show the positions of different transitions in both (a) and (b): Black downward arrows denote $T_{\mathrm{N}}$; the violet upward arrows denote $T_{\mathrm{M}}$; the green (or magenta) arrows show the field-induced transitions below (or above) $T_{\mathrm{N}}$. The inset of panel (a) enlarges the range in the dotted rectangle.

detected. As shown in Fig. 5(a), for $H \| c$ the broad peak at $T_{\mathrm{N}}$ and the small cusp in $\rho(T)$ at $T_{\mathrm{M}}$ are both suppressed monotonically to lower temperatures, and the latter disappears at around $3 \mathrm{~T}$. At higher fields an abrupt jump appears, and the field dependence of the anomaly is consistent with that found in $\chi(T)$ for the same field direction. For $H \geqslant 6 \mathrm{~T}$, broad humps are observed in $\rho(T)$ below $8 \mathrm{~K}$, as shown by the dotted upward arrow. However, a lack of a well-defined corresponding anomaly in $\chi(T)$ and $C(T) / T$ [dotted upward arrow in Figs. 4(a) and 5(c)] is observed, indicating that this may correspond to a crossover to the spin-polarized state. For $H \geqslant 3 \mathrm{~T}$ along the $c$ axis, the small peaks in $C(T) / T$ at $T_{\mathrm{M}}$ disappear while another sharp peak is observed (vertical green arrows), which has an asymmetric shape with a tail on the low-temperature side. This is assigned to be a field-induced transition, which disappears in fields above $6 \mathrm{~T}$, being consistent with $\chi(T, H)$ and $\rho(T, H)$. As shown in Fig. 5(c), all the data curves of $C(T) / T$ display a broad hump at around $3 \mathrm{~K}$. This feature is frequently observed in other Eu-based compounds with divalent Eu and is generally ascribed to the Zeeman splitting of the $J=7 / 2$ multiplet of the $\mathrm{Eu}^{2+}$ ions under internal and external magnetic fields [33-35].

For $H \perp c$ shown in Fig. 5(b), in addition to the broad peak at $T_{\mathrm{N}}$ and the small hump at $T_{\mathrm{M}}$ (suppressed for $H \geqslant 3 \mathrm{~T}$ ), a weak but discernible slope change is observed at temperatures above $T_{\mathrm{N}}$ in $\rho(T)$ for $H \geqslant 4 \mathrm{~T}$, the position of which slowly shifts to lower temperature with increasing field. On the other hand, a clear sharp drop is observed at lower temperatures in the field range of 6.0-7.5 $\mathrm{T}$ (upward green arrows), which is assigned to be another field-induced transition for $H \perp c$. Note that all these transitions are also manifested in $C(T) / T$, as shown in Fig. 5(d). $C(T) / T$ shows an increase on cooling prior to an abrupt jump at $T_{\mathrm{N}}$. This gradual increase is further enhanced under magnetic fields, which is likely due to shortrange correlations or fluctuations. The overall behavior for $H \leqslant 3 \mathrm{~T}$ is similar to that for $H \| c$; however, for $H \geqslant 4 \mathrm{~T}$, $C(T) / T$ shows two steps on cooling. From comparing the data of $C(T) / T$ with $\rho(T, H)$ and $\chi(T, H)$, it can be seen that the higher temperature step corresponds to a field-induced transition above $T_{\mathrm{N}}$ (magenta arrows), while the lower temperature step is the transition at $T_{\mathrm{N}}$, which becomes broader in higher fields. For $H=6.0-7.5 \mathrm{~T}$, an asymmetric peak is observed in $C(T) / T$ (upward green arrow), which should correspond to the field-induced transition below $T_{\mathrm{N}}$.

The isothermal magnetization $M(H)$ and field dependence of the resistivity $\rho(H)$ for $H \| c$ and $H \perp c$ are displayed in Fig. 6. Note that in all main panels, the results for both ramping up [0-9 T, solid lines] and ramping down processes [9-0 $\mathrm{T}$, broken lines] are displayed, where the data at the lowest temperature were measured first after cooling from $300 \mathrm{~K}$ in zero field, and the data at higher temperatures were subsequently measured. In $M(H)(H \| c)$, four anomalies can be resolved in the data measured on ramping up the field at $2.1 \mathrm{~K}$ : A weak slope change with broad hysteresis at $H_{\mathrm{II}}=$ $3.0 \mathrm{~T}$, steplike increases at $H_{\mathrm{III}}=3.9 \mathrm{~T}$ and $H_{\mathrm{IV}}=6.0 \mathrm{~T}$ and a kink at $H_{\mathrm{S}}=8.2 \mathrm{~T}$, above which $M(H)$ is saturated at $7 \mu_{B} / \mathrm{Eu}$, the saturated moment expected for fully polarized $\mathrm{Eu}^{2+}$ spins with $J=7 / 2$. Near $H_{\text {III }}, M(H)$ jumps by approximately $1 \mu_{B} / \mathrm{Eu}$ and then linearly increases with field. The extrapolation of this linear part to $H=0$ yields a finite intercept. This is generally an indication of a spin-flip transition [36]. The same features occur at $H_{\mathrm{IV}}$, where the magnetization jump is about $2 \mu_{B} / \mathrm{Eu}$. At temperatures above $7.5 \mathrm{~K}$ but below $15 \mathrm{~K}$, only one transition can be found in $M(H)$, which shows a relatively broad anomaly labeled $H_{\mathrm{I}}$ in Fig. 6(a). For $T \geqslant 15 \mathrm{~K}, M(H)$ shows a sublinear field dependence with no detectable anomaly. All these transitions can also be observed in the isothermal $\rho(H)(H \| c)$ data at the corresponding temperatures, as shown in Fig. 6(c). For $T \leqslant$ $7.5 \mathrm{~K}$, the $\rho(H)(H \| c)$ curves display a kink with a broad hysteresis loop in low fields, which then abruptly jumps (by about $4 \mu \Omega$-cm for $1.9 \mathrm{~K}$ ) at $H_{\mathrm{III}}$, flattens, and subsequently drops (by about $10 \mu \Omega$-cm for $1.9 \mathrm{~K}$ ) at $H_{\mathrm{IV}}$. After that, the data decrease slowly with increasing field and bend at $H_{\mathrm{S}}$. At $7.5 \mathrm{~K} \leqslant T \leqslant 15 \mathrm{~K}, \rho(H)(H \| c)$ exhibits a broad drop at fields corresponding to $H_{\mathrm{I}}$.

For $H \perp c$, two transitions are observed in $M(H)$ at $2 \mathrm{~K}$ : One at $3 \mathrm{~T}$ [labeled as $H_{\mathrm{II}}$ ] showing a weak slope change and another at $6.0 \mathrm{~T}$ [labeled as $H_{\mathrm{III}^{\prime}}$ ] showing a sharp change of slope together with a hysteresis extending from 2.5 to $6.1 \mathrm{~T}$. Consistently, $\rho(H)(H \perp c)$ [Fig. 6(d)] displays two steplike decreases at $H_{\mathrm{II}}$ and $H_{\mathrm{III}}$ with sizable hysteresis loops. With increasing temperature, $H_{\mathrm{II}}\left(H_{\mathrm{III}}\right)$ shifts to lower (higher) fields. Above $5 \mathrm{~K}, \rho(H)(H \perp c)$ displays a single broad drop 

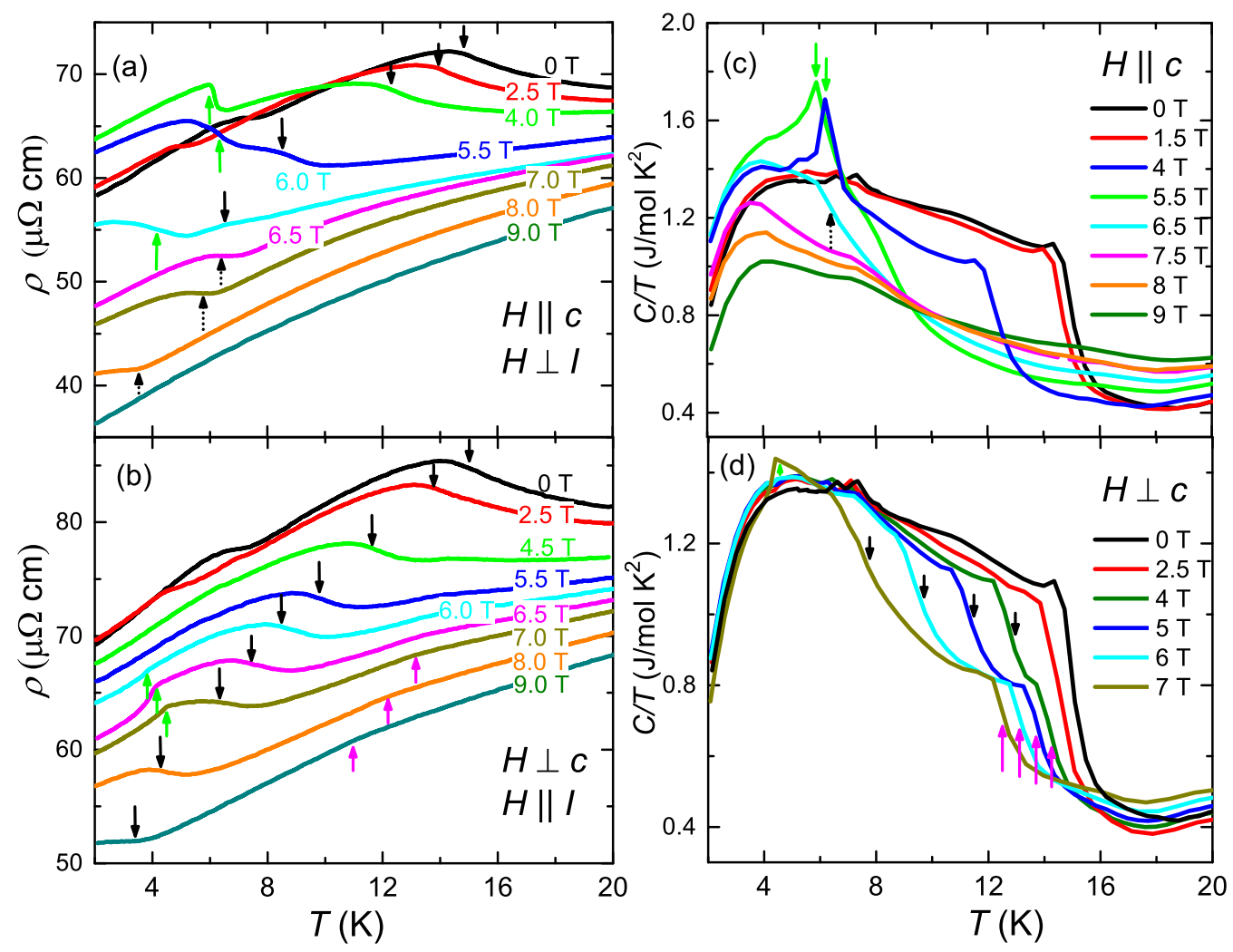

FIG. 5. (a), (b) Temperature dependence of the resistivity $\rho(T)$, and (c), (d) specific heat $C(T) / T$ in constant magnetic fields up to 9 T applied parallel and perpendicular to the $c$ axis, respectively. The arrows with different colors are used to mark different phase transitions, which are labeled in the same manner as those in Fig. 4.
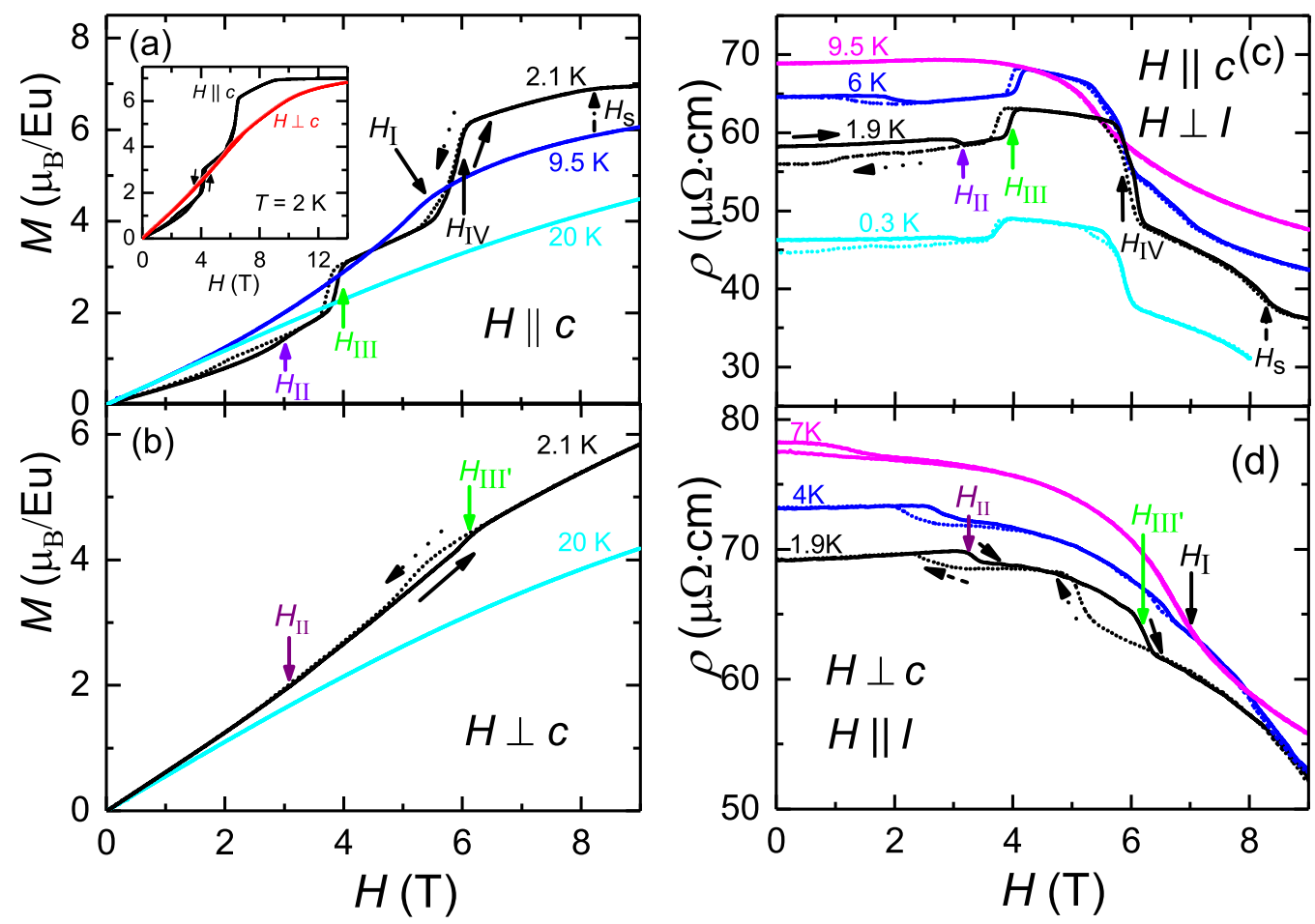

FIG. 6. Isothermal magnetization $M(H)$ measured at several temperatures for (a) $H \| c$ and (b) $H \perp c$. The field dependence of the electrical resistivity $\rho(H)$ is also displayed for (c) $H \| c$ and (d) $H \perp c$. The inset in (a) shows the $M(H)$ curves at $2 \mathrm{~K}$ with fields up to $14 \mathrm{~T}$. Only the $\rho(H)$ data at $T=0.3 \mathrm{~K}$ in (c) are shifted. In all the figures, the vertical arrows show the positions of transitions, for which the same colors are used as in Figs. 4 and 5. The directions of the field ramping are indicated by tilted arrows. 

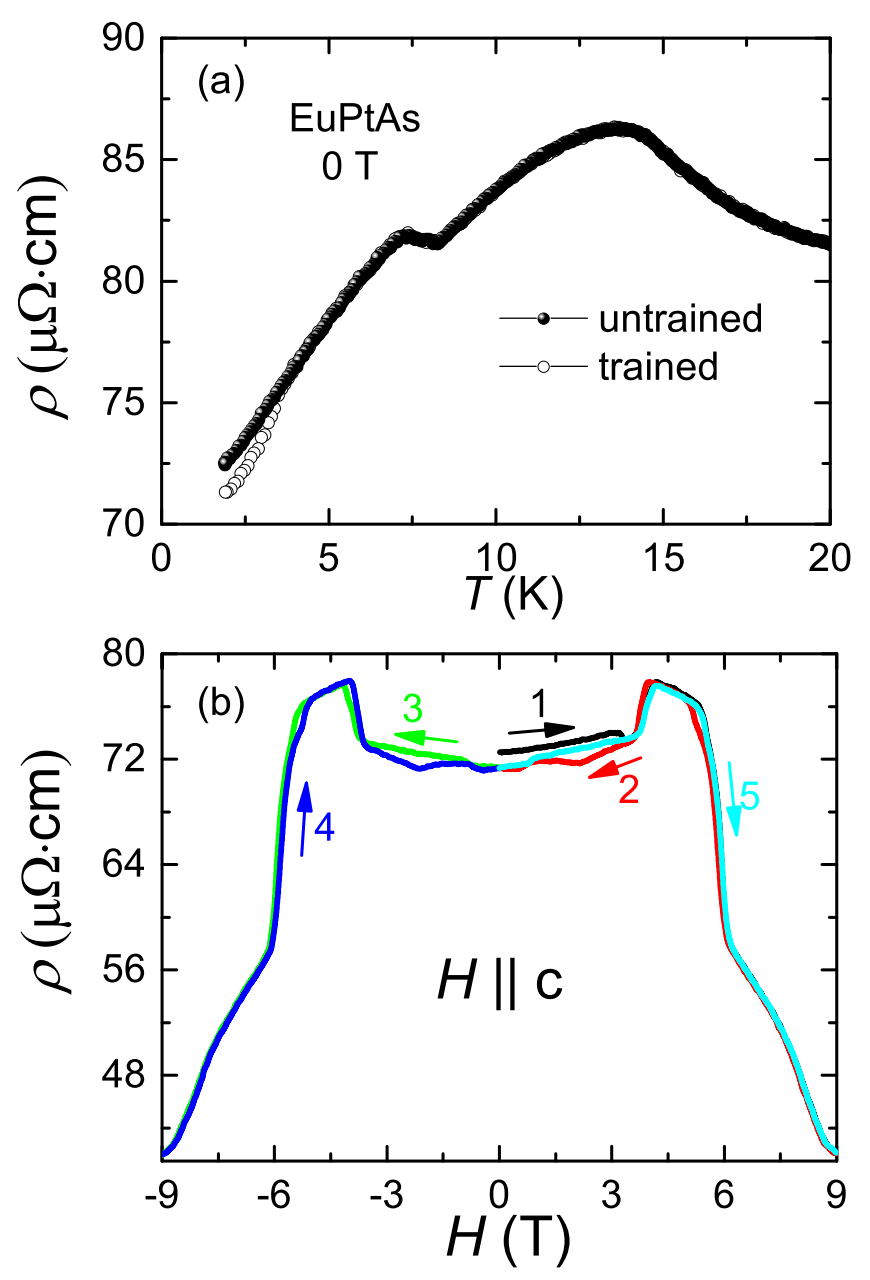

FIG. 7. The effects of cooling history on the resistivity: (a) A comparison of $\rho(T)$ at zero field for trained and untrained cases, as described in the text. (b) $\rho(H)$ measured after cooling from $300 \mathrm{~K}$ in zero field. The numbers and arrows denote the order in which the measurements were performed and the directions of the field scans, respectively.

(labeled as $H_{\mathrm{I}}$ ) at around $7 \mathrm{~T}$ for $T=7 \mathrm{~K}$. It can be seen below that the temperature dependence of $H_{\mathrm{I}}$ and $H_{\mathrm{II}}$ corresponds to the field dependence of $T_{\mathrm{N}}$ and $T_{\mathrm{M}}$, respectively. Magnetization measurements have been further performed up to $14 \mathrm{~T}$ at $2 \mathrm{~K}$. As shown in the inset of Fig. 6(a), the $M(H)$ curves show no additional anomalies at higher fields for either field direction. Note that for fields applied parallel to the $c$ axis, the magnetization saturates at $H_{\mathrm{S}}$ with a moment of $7 \mu_{B} / \mathrm{Eu}$, while perpendicular to the $c$ axis there is no full saturation even up to $14 \mathrm{~T}$.

Note that at 0.3 and $1.9 \mathrm{~K}$, the broad hysteresis loop in $\rho(H)(H \| c)$ does not close when the field is reduced down to $0 \mathrm{~T}$, while no zero-field hysteresis or remnant magnetization is observed in $M(H)(H \| c)$ in Fig. 6(a). We further found that the hysteresis near zero field is only observed in $\rho(H)(H \| c)$ between the initial field scan after zero-field cooling and the subsequent field sweeps, as shown in Fig. 7(b). On the other hand, the hysteresis at zero field also disappears if the sample is cooled in a field of $9 \mathrm{~T}$ and then measured on ramping down to zero and back up to $9 \mathrm{~T}$. To further confirm the influence of cooling history on the zero-field resistivity, we have measured $\rho(T)$ by adopting two different cooling processes: (1) The sample is cooled in zero field from 20 to $1.9 \mathrm{~K}$, and then the $\rho(T)$ is measured on warming; (2) the sample is cooled in an applied field of $9 \mathrm{~T}$ from 20 to $1.9 \mathrm{~K}$, and the field is then decreased to zero before measuring $\rho(T)$ on warming. Differences are expected for $\rho(T)$ measured by different processes when there are multiple magnetic domains in the sample, which has been illustrated in a number of compounds, such as ferromagnetic domains in $\mathrm{SrRuO}_{3}$ [37] and MnP [38], all-in-all-out type AFM domains in $\mathrm{Nd}_{2} \mathrm{Ir}_{2} \mathrm{O}_{7}$ [39], and competing domains in $\mathrm{EuCuSb}$ [40]. In these multidomain cases, the above-mentioned process (1) was termed as measuring in the untrained state (multidomain state), while process (2) was termed as measured in the trained state (domain-aligned state). Our $\rho(T)$ data about EuPtAs are shown in Fig. 7(a), where a negative magnetoresistance is observed after the training processes, being consistent with that observed in $\rho(H)(H \| c)$ shown in Fig. 7(b). This observation is similar to that in $\mathrm{SrRuO}_{3}$ and $\mathrm{MnP}$ with multiple ferromagnetic domains [37,38], where the alignment of the magnetic domains along the field direction in the trained state leads to a reduction of the resistivity. This is in contrast to that reported for $\mathrm{Nd}_{2} \mathrm{Ir}_{2} \mathrm{O}_{7}$ and $\mathrm{EuCuSb}$, which both show a positive magnetoresistance $[39,40]$, where it was proposed that $\mathrm{Nd}_{2} \operatorname{Ir}_{2} \mathrm{O}_{7}$ hosts metallic domain walls due to the nontrivial topology $[39,41]$, while in $\mathrm{EuCuSb}$, the applied field may preferentially select for the domain with lower forming energy but higher resistance [40]. As such, the negative magnetoresistance on applying a training field gives evidence for multiple magnetic domains. While the absence of remnant magnetization in zero field suggests that these are AFM domains, confirmation of their nature requires the microscopic characterization of the magnetic structure.

\section{C. $H-T$ phase diagrams}

Based on all the data displayed above, we constructed the $H-T$ phase diagrams for the two field directions. As shown in Fig. 8, transition points from different measurements coincide well with each other. The phase diagrams for $H \| c$ and $H \perp c$ show some similarities. For example, both $T_{\mathrm{N}}$ and $T_{\mathrm{M}}$ are monotonically suppressed to lower temperature, and a field-induced phase (phase III and phase III') appears below $T_{\mathrm{N}}$. The suppression rates of $T_{\mathrm{N}}(H)(H \| c)$ and $T_{\mathrm{N}}(H)(H \perp c)$ are comparable for $H \leqslant 5 \mathrm{~T}$, suggesting weak magnetocrystalline anisotropy. This is consistent with the small single-ion anisotropy expected for $\mathrm{Eu}^{2+}$, where the Hund's rule ground state lacks an orbital moment $(L=0)$.

Differences between these two phase diagrams are also apparent. For $H \| c, T_{\mathrm{N}}$ is abruptly suppressed at around $6 \mathrm{~T}$, above which a phase IV appears at $H \geqslant 6 \mathrm{~T}$ located between phase III and the field-polarized phase, where phase III has a dome shape below $T_{\mathrm{N}}$, while for $H \perp c, T_{\mathrm{N}}$ is suppressed considerably more slowly for $H \geqslant 6 \mathrm{~T}$, exhibiting a long tail stretching to higher fields. The field-induced transition (solid green line) appears to terminate on intersecting $T_{\mathrm{N}}(H)$ at around $5 \mathrm{~K}$, which, together with $T_{\mathrm{N}}(H)$, becomes the boundary of the field-induced phase III'. On the other hand, at higher fields there is evidence for a field-induced phase IV' 


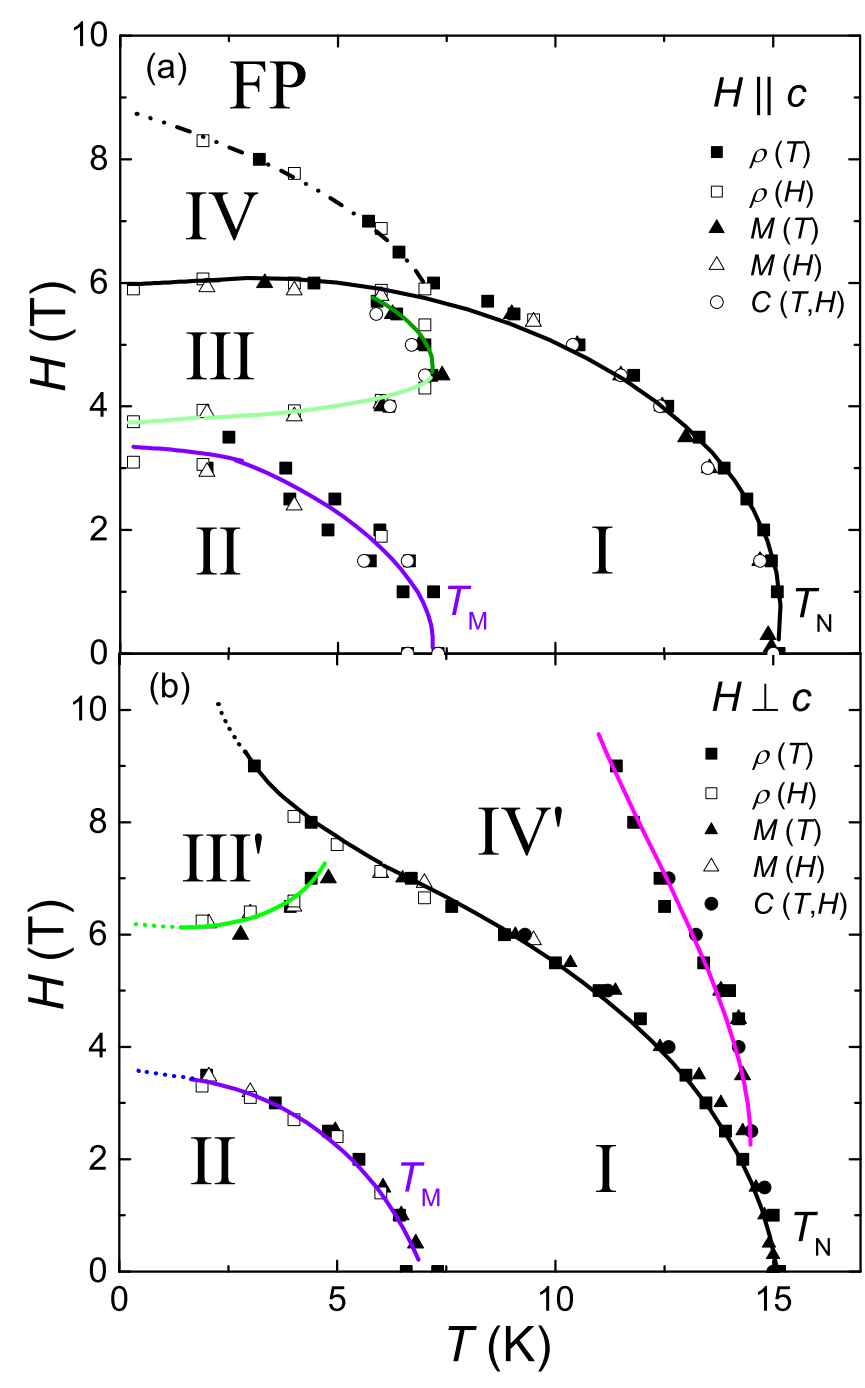

FIG. 8. $T-H$ phase diagrams for EuPtAs, constructed from measurements of $\rho(T, H), C(T, H)$, and $\chi(T, H)$. The solid lines are guides to the eyes, while the colors correspond to the arrows labeling the transitions in Figs. 4-6. The boundary of phase III in (a) is marked with both dark and light green, which correspond to slight differences in the anomalies observed in the respective field ranges. The Roman numerals label the different magnetic phases.

appearing above $T_{\mathrm{N}}$, and on cooling for $H \geqslant 7 \mathrm{~T}$, there is a transition directly from this phase to phase III' $^{\prime}$. The rather complex phase diagrams could be a result of the presence of both Heisenberg-type magnetic exchange interactions and the DM interaction, as have been observed in other noncentrosymmetric compounds [6,14,18].

There are some notable features of the field-induced phases III and phase III'. In the temperature-dependent measurements, on cooling through the transition into phase III: $\rho(T)$ has a steplike increase under applied fields of 4-6 T, while $\chi(T)$ displays a jump with thermal hysteresis in fields of 4-4.6 $\mathrm{T}$ but a drop with thermal hysteresis in the field range of 4.6-6 T, both exhibiting a rather linear behavior after cooling across the transition; $C(T) / T$ shows a sharp asymmetric peak without hysteresis and exhibits a tail on the lower temperature side. In the field-dependent measurements, $\rho(H)$ shows an abrupt increase at the lower field phase boundary while displaying a larger drop at the higher field phase boundary with sizable hysteresis at both phase boundaries. $M(H)$ at $2.1 \mathrm{~K}$ jumps by approximately $1 \mu_{\mathrm{B}} / \mathrm{Eu}$ at the lower boundary and approximately $2 \mu_{\mathrm{B}} / \mathrm{Eu}$ at the higher field boundary, but increases linearly in between. On the other hand, on cooling into phase $\mathrm{III}^{\prime}, \rho(T)$ exhibits a small but sharp drop, while a sharp increase is observed in $\chi(T)$ followed by a flat plateau at $6 \mathrm{~T}$. In $C(T) / T$, there is an asymmetric peak on entering phase III'. $\rho(H)$ undergoes a smaller drop on crossing the lower boundary of phase $\mathrm{III}^{\prime}$, while $M(H)$ shows a sharp change of slope (together with hysteresis) and increases sublinearly inside phase III'.

We are not able to determine the magnetic structures of these different phases solely from our present measurements of bulk properties. However, it is of particular interest to compare the phase diagrams of EuPtAs with those of EuPtSi and $\mathrm{CeAlGe}$, both of which crystallize in noncentrosymmetric structures with the latter being isostructural to EuPtAs. EuPtSi shows a phase diagram that is typical for chiral B20 compounds when the field is applied along the crystalline [111] direction, where the skyrmion-hosting A phase arises out of the low-field single- $k$ helimagnetic phase $[14,15,42]$. Notably the A phase in EuPtSi has a fully closed phase boundary, existing only in limited temperature and field ranges in the equilibrium conditions, although a metastable A phase can extend down to $60 \mathrm{mK}$ under field cooling [43]. While for $\mathrm{CeAlGe}$, the $T_{\mathrm{N}}$ of $4.4 \mathrm{~K}$ is continuously suppressed to lower temperature, and two field-induced transitions below $T_{\mathrm{N}}$ are observed, which descend nearly parallel to each other to lower temperature with increasing field, meaning that the boundary of the nontrivial merons/antimeron phase is open on the low-temperature side, likely extending to zero temperature [19]. This is similar to the case for centrosymmetic $\mathrm{Gd}_{2} \mathrm{PdSi}_{3}$, where the skyrmion phase also lacks a boundary on the low-temperature side [20]; here, $\rho(H)$ shows abrupt increases and decreases on either side of the skyrmion phase boundary [44], which is similar to those observed for phase III in Fig. 6(c).

In order to microscopically characterize the magnetic structure of the different phases and to look for evidence of topologically nontrivial spin textures (especially, in phase III), it is necessary to both look for the presence of a topological Hall effect and to perform neutron diffraction or magnetic x-ray scattering studies, which are currently underway.

\section{SUMMARY}

In summary, we have synthesized single crystals of the noncentrosymmetric Eu-based compound EuPtAs and studied the physical properties by measuring resistivity, heat capacity, and magnetic susceptibility. In zero field, two transitions are found with a second-order AFM transition at $T_{\mathrm{N}}=14.8 \mathrm{~K}$ and a first-order transition at $T_{\mathrm{M}}=7.5 \mathrm{~K}$, which were evidenced to be arising from well-localized $4 f$ electronic states of $\mathrm{Eu}^{2+}$.

Measurements were performed in applied magnetic fields up to $9 \mathrm{~T}$ parallel and perpendicular to the $c$ axis, which allow us to construct the phase diagrams for both field 
directions. Our data show that both $T_{\mathrm{N}}$ and $T_{\mathrm{M}}$ are continuously suppressed by fields for both $H \| c$ and $H \perp c$, and there are multiple field-induced transitions for both directions, either above or below $T_{\mathrm{N}}(H)$, including a dome-shaped phase III for $H \| c$. Furthermore, the resistivity measurements under different cooling processes show clear evidence for multiple magnetic domains in the zero-field phase. Our study shows the presence of complex temperature-field phase diagrams for EuPtAs, with two zero-field transitions and multiple field-induced phases. In order to characterize the microscopic nature of the magnetism in EuPtAs, it is of particular interest to determine the magnetic structures using neutron or x-ray scattering.

\section{ACKNOWLEDGMENTS}

This work was supported by the National Key R\&D Program of China (No. 2017YFA0303100 and No. 2016YFA0300202), the National Natural Science Foundation of China (No. 12034017 and No. 11974306), the Key R\&D Program of Zhejiang Province, China (2021C01002), and the Fundamental Research Funds for the Central Universities. We thank T. Shang for fruitful discussions, who acknowledges support from the Natural Science Foundation of Shanghai (Grants No. 21ZR1420500 and No. 21JC1402300). W. Xie thanks support from the committee of SCES2019 (Okayama, Japan).
[1] I. Dzyaloshinsky, J. Phys. Chem. Solids 4, 241 (1958).

[2] T. Moriya, Phys. Rev. 120, 91 (1960).

[3] N. Nagaosa and Y. Tokura, Nat. Nanotechnol. 8, 899 (2013).

[4] E. Y. Ishikawa, K. Tajima, D. Bloch, and M. Roth, Solid State Commun. 19, 525 (1976).

[5] C. Pappas, E. Lelièvre-Berna, P. Falus, P. M. Bentley, E. Moskvin, S. Grigoriev, P. Fouquet, and B. Farago, Phys. Rev. Lett. 102, 197202 (2009).

[6] S. Mühlbauer, B. Binz, F. Jonietz, C. Pfleiderer, A. Rosch, A. Neubauer, R. Gergii, and P. Böni, Science 323, 915 (2009).

[7] X. Z. Yu, Y. Onose, N. Kanazawa, J. H. Park, J. H. Han, Y. Matsui, N. Nagaosa, and Y. Tokura, Nature (London) 465, 901 (2010).

[8] W. Münzer, A. Neubauer, T. Adams, S. Mühlbauer, C. Franz, F. Jonietz, R. Georgii, P. Böni, B. Pedersen, M. Schmidt, A. Rosch, and C. Pfleiderer, Phys. Rev. B 81, 041203(R) (2010).

[9] M. Uchida, N. Nagaosa, J. P. He, Y. Kaneko, S. Iguchi, Y. Matsui, and Y. Tokura, Phys. Rev. B 77, 184402 (2008).

[10] H. Wilhelm, M. Baenitz, M. Schmidt, U. K. Rossler, A. A. Leonov, and A. N. Bogdanov, Phys. Rev. Lett. 107, 127203 (2011)

[11] S. Seki, X. Z. Yu, and S. Ishiwata, Science 336, 198 (2012).

[12] T. Adams, A. Chacon, M. Wagner, A. Bauer, G. Brandl, B. Pedersen, H. Berger, P. Lemmens, and C. Pfleiderer, Phys. Rev. Lett. 108, 237204 (2012).

[13] J. Evers and G. Oehlinger, J. Solid State Chem. 62, 133 (1986).

[14] M. Kakihana, D. Aoki, A. Nakamura, F. Honda, M. Nakashima, Y. Amako, S. Nakamura, T. Sakakibara, M. Hedo, T. Nakama, and Y. Ōnuki, J. Phys. Soc. Jpn. 87, 023701 (2018).

[15] K. Kaneko, M. D. Frontzek, M. Matsuda, A. Nakao, K. Munakata, T. Ohhara, M. Kakihana, Y. Haga, M. Hedo, T. Nakama, and Y. Ōnuki, J. Phys. Soc. Jpn. 88, 013702 (2018).

[16] S. Hayami and R. Yambe, J. Phys. Soc. Jpn. 90, 073705 (2021).

[17] M. Kakihana, D. Aoki, A. Nakamura, F. Honda, M. Nakashima, Y. Amako, T. Takeuchi, H. Harima, M. Hedo, T. Nakama, and Y. Ōnuki, J. Phys. Soc. Jpn. 88, 094705 (2019).

[18] I. Kézsmárki, S. Bordács, P. Milde, E. Neuber, L. M. Eng, J. S. White, H. M. Rønnow, C. D. Dewhurst, M. Mochizuki, K. Yanai, H. Nakamura, D. Ehlers, V. Tsurkan, and A. Loidl, Nat. Mater. 14, 1116 (2015).

[19] P. Puphal, V. Pomjakushin, N. Kanazawa, V. Ukleev, D. J. Gawryluk, J. Ma, M. Naamneh, N. C. Plumb, L. Keller, R. Cubitt, E. Pomjakushina, and J. S. White, Phys. Rev. Lett. 124, 017202 (2020).
[20] T. Kurumaji, T. Nakajima, M. Hirschberger, A. Kikkawa, Y. Yamasaki, H. Sagayama, H. Nakao, Y. Taguchi, T. Arima, and Y. Tokura, Science 365, 914 (2019).

[21] N. D. Khanh, T. Nakajima, X. Yu, S. Gao, K. Shibata, M. Hirschberger, Y. Yamasaki, H. Sagayama, H. Nakao, L. Peng, K. Nakajima, R. Takagi, T. Arima, Y. Tokura, and S. Seki, Nat. Nanotechnol. 15, 444 (2020).

[22] M. Hirschberger, T. Nakajima, S. Gao, L. Peng, A. Kikkawa, T. Kurumaji, M. Kriener, Y. Yamasaki, H. Sagayama, H. Nakao, K. Ohishi, K. Kakurai, Y. Taguchi, X. Yu, T. Arima, and Y. Tokura, Nat. Commun. 10, 5831 (2019).

[23] T. Nomoto, T. Koretsune, and R. Arita, Phys. Rev. Lett. 125, 117204 (2020).

[24] S. Gupta and K. G. Suresh, J. Alloys Compd. 618, 562 (2015).

[25] G. Wenski and A. Mewis, Z. Anorg. Alleg. Chem. 543, 49 (1986).

[26] R. Müllmann, U. Ernet, B. D. Mosel, H. Eckert, R. K. Kremer, R.-D. Hoffmann, and R. Pöttgen, J. Mater. Chem. 11, 1133 (2001).

[27] O. Bednarchuk, A. Gagor, and D. Kaczorowski, J. Alloys Compd. 622, 432 (2015).

[28] N. F. Mott and H. Jones, The Theory of the Properties of Metals and Alloys (Oxford University Press, Oxford, UK, 1958).

[29] V. K. Anand and D. C. Johnston, Phys. Rev. B 94, 014431 (2016).

[30] P. Zhang, H. F. Zhai, Y. P. Li, Z. Wang, J. Chen, S. Y. Zheng, Q. Tao, J. H. Dai, G. H. Cao, and Z. A. Xu, J. Alloys Compd. 728, 959 (2017).

[31] V. K. Anand and D. C. Johnston, J. Phys.: Condens. Matter 26, 286002 (2014).

[32] A. Maurya, P. Bonville, R. Kulkarni, A. Thamizhavel, and S. K. Dhar, J. Magn. Magn. Mater. 401, 823 (2016).

[33] W. B. Jiang, M. Smidman, W. Xie, J. Y. Liu, J. M. Lee, J. M. Chen, S. C. Ho, H. Ishii, K. D. Tsuei, C. Y. Guo, Y. J. Zhang, Hanoh Lee, and H. Q. Yuan, Phys. Rev. B 95, 024416 (2017).

[34] S. Süllow, I. Prasad, M. C. Aronson, J. L. Sarrao, Z. Fisk, D. Hristova, A. H. Lacerda, M. F. Hundley, A. Vigliante, and D. Gibbs, Phys. Rev. B 57, 5860 (1998).

[35] V. K. Anand and D. C. Johnston, Phys. Rev. B 91, 184403 (2015).

[36] S. Blundell, Magnetism in Condensed Matter (Oxford University Press, New York, 2001). 
[37] L. Klein, Y. Kats, A. F. Marshall, J. W. Reiner, T. H. Geballe, M. R. Beasley, and A. Kapitulnik, Phys. Rev. Lett. 84, 6090 (2000).

[38] N. Jiang, Y. Nii, H. Arisawa, E. Saitoh, J. Ohe, and Y. Onose, Phys. Rev. Lett. 126, 177205 (2021).

[39] K. Ueda, J. Fujioka, B.-J. Yang, J. Shiogai, A. Tsukazaki, S. Nakamura, S. Awaji, N. Nagaosa, and Y. Tokura, Phys. Rev. Lett. 115, 056402 (2015).

[40] H. Takahashi, K. Aono, Y. Nambu, R. Kiyanagi, T. Nomoto, M. Sakano, K. Ishizaka, R. Arita, and S. Ishiwata, Phys. Rev. B 102, 174425 (2020).
[41] K. Ueda, J. Fujioka, Y. Takahashi, T. Suzuki, S. Ishiwata, Y. Taguchi, M. Kawasaki, and Y. Tokura, Phys. Rev. B 89, 075127 (2014).

[42] C. Tabata, T. Matsumura, H. Nakao, S. Michimura, M. Kakihana, T. Inami, K. Kaneko, M. Hedo, T. Nakama, and Y. Ōnuki, J. Phys. Soc. Jpn. 88, 093704 (2019).

[43] T. Sakakibara, S. Nakamura, S. Kittaka, M. Kakihana, M. Hedo, T. Nakama, and Y. Ōnuki, J. Phys. Soc. Jpn. 90, 064701 (2021).

[44] H. Zhang, Q. Huang, L. Hao, J. Yang, K. Noordhoek, S. Pandey, H. Zhou, and J. Liu, New J. Phys. 22, 083056 (2020). 